\title{
CALIBRATION PROCEDURES ON OBLIQUE CAMERA SETUPS
}

\author{
G.Kemper ${ }^{\mathrm{a}}$, B. Melykuti ${ }^{\mathrm{b}}, \mathrm{C} . \mathrm{Yu}^{\mathrm{c}}{ }^{*}$ \\ a GGS GmbH,Speyer / Germany - kemper@ggs-speyer.de

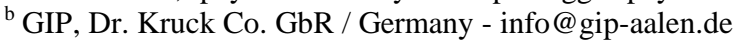 \\ ${ }^{\mathrm{c}}$ Link Fast Ltd. / Taiwan - carol_yu@linkfast.com.tw
}

\section{Commission I, WG I/3}

KEY WORDS: Oblique Images, Camera-calibration, 3D City-model, Multi-sensor, Boresight-calibration

\begin{abstract}
:
Beside the creation of virtual animated 3D City models, analysis for homeland security and city planning, the accurately determination of geometric features out of oblique imagery is an important task today. Due to the huge number of single images the reduction of control points force to make use of direct referencing devices. This causes a precise camera-calibration and additional adjustment procedures.

This paper aims to show the workflow of the various calibration steps and will present examples of the calibration flight with the final 3D City model. In difference to most other software, the oblique cameras are used not as co-registered sensors in relation to the nadir one, all camera images enter the AT process as single pre-oriented data. This enables a better post calibration in order to detect variations in the single camera calibration and other mechanical effects.

The shown sensor (Oblique Imager) is based o 5 Phase One cameras were the nadir one has 80 MPIX equipped with a $50 \mathrm{~mm}$ lens while the oblique ones capture images with $50 \mathrm{MPix}$ using $80 \mathrm{~mm}$ lenses. The cameras are mounted robust inside a housing to protect this against physical and thermal deformations. The sensor head hosts also an IMU which is connected to a POS AV GNSS Receiver. The sensor is stabilized by a gyro-mount which creates floating Antenna -IMU lever arms. They had to be registered together with the Raw GNSS-IMU Data.

The camera calibration procedure was performed based on a special calibration flight with 351 shoots of all 5 cameras and registered the GPS/IMU data. This specific mission was designed in two different altitudes with additional cross lines on each flying heights. The five images from each exposure positions have no overlaps but in the block there are many overlaps resulting in up to 200 measurements per points. On each photo there were in average 110 well distributed measured points which is a satisfying number for the camera calibration. In a first step with the help of the nadir camera and the GPS/IMU data, an initial orientation correction and radial correction were calculated. With this approach, the whole project was calculated and calibrated in one step. During the iteration process the radial and tangential parameters were switched on individually for the camera heads and after that the camera constants and principal point positions were checked and finally calibrated.

Besides that, the bore side calibration can be performed either on basis of the nadir camera and their offsets, or independently for each camera without correlation to the others. This must be performed in a complete mission anyway to get stability between the single camera heads. Determining the lever arms of the nodal-points to the IMU centre needs more caution than for a single camera especially due to the strong tilt angle.

Prepared all these previous steps, you get a highly accurate sensor that enables a fully automated data extraction with a rapid update of you existing data. Frequently monitoring urban dynamics is then possible in fully $3 \mathrm{D}$ environment.
\end{abstract}

\section{INTRODUCTION / MOTIVATION}

Oblique imaging became very popular within the last years even that way of capturing images was already used since many decades. Besides architectural oblique imaging, also for open mining and other special tasks oblique images were used for a long time. About 8 years ago, there was a higher request for such data, either for homeland-security issues or for internet applications as Bing, Virtual Earth, Google, city planning and others. With some exceptions, a core georeference to display the data on the approximate place over a map was sufficient. Besides that, the image quality and resolution of the cameras was poor that time. The use of such data was situated in specific segments only.

Today we make use of cameras with medium format sensors that capture images with 50-100 MPIX. Not the higher resolution only, also the precise image geometry of such mapping cameras widened the range of using these data for precise mapping and modelling too. Cameras mounted between 30 and 50 degrees off nadir are typically defined as oblique.
The use of the today's captured oblique images are in the field of precise 3D City models, to generate high dense DSMs with a extreme good high accuracy and a high redundancy of image data in order to texture the facades. Since 2 years, this forced a growing request for such setups. It sounds not to be complicate to mount 5 medium format cameras together but to design an accurate and stabile system that supports direct georeferencing needs much more than a simple joint camera platform. To build up a stable and well calibrated system, this paper aims to show which steps are made to get a most sufficient system implemented.

\section{HARDWARE DESIGN / ELEMENT}

Linkfast Company in Taiwan requested for a precise an Oblique camera-system at GGS and the development started beginning of 2015. The intention was to build a high resolution, reliable, robust and precise oblique sensor ready calibrated to fit into a GSM4000 mount and to be connected to a POS-AV system for $3 \mathrm{D}$ city mapping in Taiwan. 
The heart of any imaging sensor is the camera. High resolutions mid format mapping cameras have to have a high quality lenses supporting the resolution of the sensor. This lens has to have a high quality to be geometrically calibrated. There is also a must of having a central leaf shutter to prevent time delayed distortions (as with a focal plane shutter). A solid mounting of the lens at the camera body also is keyfeature in order to keep the lens calibration stabile. Then there has to be also an electrical interface to trigger the camera and to achieve a mid exposure pulse (event) on the exact time of exposure.

The first choice was the cameras that had to be precisely synchronized in order to support the GNSS-INS system. The PhaseOne Mid Format Mapping Cameras support a daisy chain solution in order to trigger the cameras precisely. That way, just one trigger signal fires all cameras simultaneously and the event is for all cameras better than $100 \mu$ s the same. The final choice was, one $80 \mathrm{MPix}$ IXA-R Camera with a $50 \mathrm{~mm}$ lens for nadir and four 50 Mpix IXU Cameras with $80 \mathrm{~mm}$ lens for a 40 degrees view.

The first job was the mechanical mounting of the cameras to get them fit into a predefined mount in order to support the mounting height for minimizing the risk of vignetting due to the camera hole in the aircraft. Thus, a variable mounting to adjusts the oblique angle with 1 degree steps and a most narrow mounting of the 5 cameras was designed to achieve a optimized compact sensor configuration. At the end, a sensor head with a $380 \mathrm{~mm}$ diameter was built based on robust Aluminum CNC manufactured layout. To get the cameras close together support both, stability in the relative camera-angles and compactness to get the cameras in a standard mounting.

The cameras support a daisy chain solution and with the Fast Sync shutters, the 5 cameras shoot within $100 \mu \mathrm{S}$ exactly at the same time. This makes need of only 1 event-port registering the mid exposure pulse to be stored in the GNSS-INS Receiver. So far the interfacing for triggering and event-registration is already implemented in the PhaseOne camera technology. Besides these 2 interfaces, the cameras control each other to make sure that all 5 cameras capture images and to ensure all are ready for the next capture. The Cameras were powered using a DC-DC device to guarantee clean $24 \mathrm{~V} \mathrm{DV}$ from the aircraft without any spikes. A industrial PC board was mounted inside the camera cone to make everything compact in order to reduce the number of cables. The data were stored on SSD drives using the cameras USB-3 connection via the IX Capture software.

For triggering the cameras, an AeroSnap device was used to change the serial information from the AeroTopoL FMS to a TTL signal.

As Mission Planning and Flight Management System AeroTopoL was implemented. The oblique planning tools assist in a proper selection of flight-lines and snap-points while the navigation mode makes use of the NMEA output of the PosAV as well as the event based data sent from the Applanix to report to AeroTopoL that an images was taken.

The GSM4000 is controlled by the INS of the PosAV. Activation and deactivating the stabilizer is made by the operator using the PosAV Controller. The raw GNNS-INS data are stored on a memory card together with the gimbal angles in order to calculate the floating lever arms.

\section{IMPLEMENTATION OF THE CALIBRATION FLIGHT}

GGS operates a testfield in the city of Speyer with a sufficient number of GCPs, a GNSS Base station and other tools to validate aerial surveying projects. The city is ideal for testing oblique images because there are important historical buildings as the roman cathedral, houses from the late mid-ages and other nice objects. Due to some higher buildings, the calibration of the lenses is good. But in addition this was improved by using 2 flight altitudes.

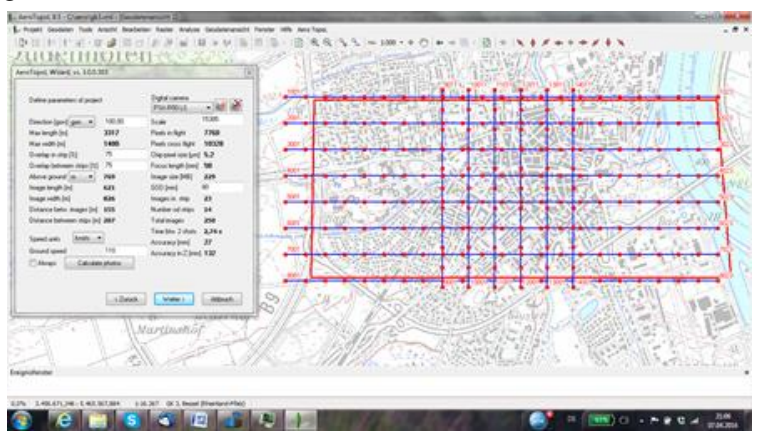

Figure 1: Mission plan of the $8 \mathrm{~cm}$ mission made with AeroTopoL FMS

The $8 \mathrm{~cm}$ mission was planned with $75 \%$ overlap and $75 \%$ sidelap. This results in $8 \mathrm{E}-\mathrm{W}$ runs. In addition there were designed 6 perpendicular strips. In total there were a number of $250 * 5$ images on this altitude.

The $14 \mathrm{~cm}$ Mission was designed with same overlap and sidelap which gave $5 \mathrm{E}-\mathrm{W}$ runs and 3 perpendicular ones have been added to them. A number of $91 * 5$ photos was the result here.

The intention of this selection was to be able to process an AT for each camera stand alone if needed. This is a bit difficult task when flying with a tilted camera east west and back. That causes for a specific camera a huge sidelap in one direction and a very poor sidelap in the other.

The Oblique Imager with the 5 sensor-heads was installed in a Cessna 206 and the flight was performed on $8^{\text {th }}$ of August 2015.

After flight, the GNSS-IMU data were processed in IE Software using the local base station. The images ran through Capture One Raw Image processing software to generate Tiff files.

\section{CALIBRATION PROCEDURE}

The first adjustment was performed in Agisoft/Photoscan in order to check if the orientation is found in the approximate position. The EO from the GNSS-IMU was used to start the tiepoint matching. This is a good reliability check to find gross errors. The selection of tie-points went straight without problems and after a cleanup of bad matches and reducing the huge amount of tie-points, the data were exported into the BINGO software.

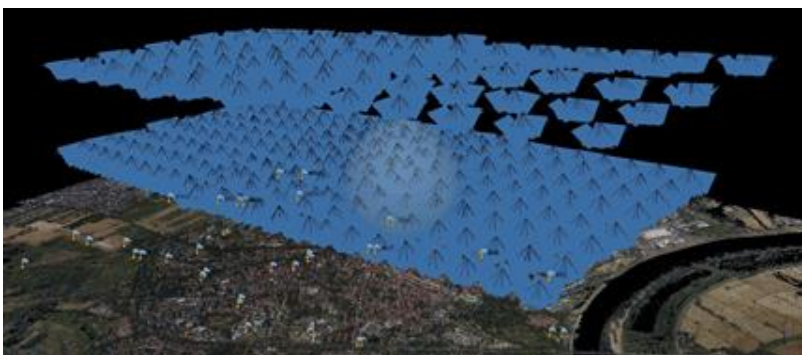

Figure 2: Results of the joint AT of all cameras and both flight altitudes 
The five images from each exposure point have not any overlap but in the block are plenty of them that result in up to 200 measurements per points. On each photo were more than 100 well distributed measured points which is a satisfying number for the camera calibration.

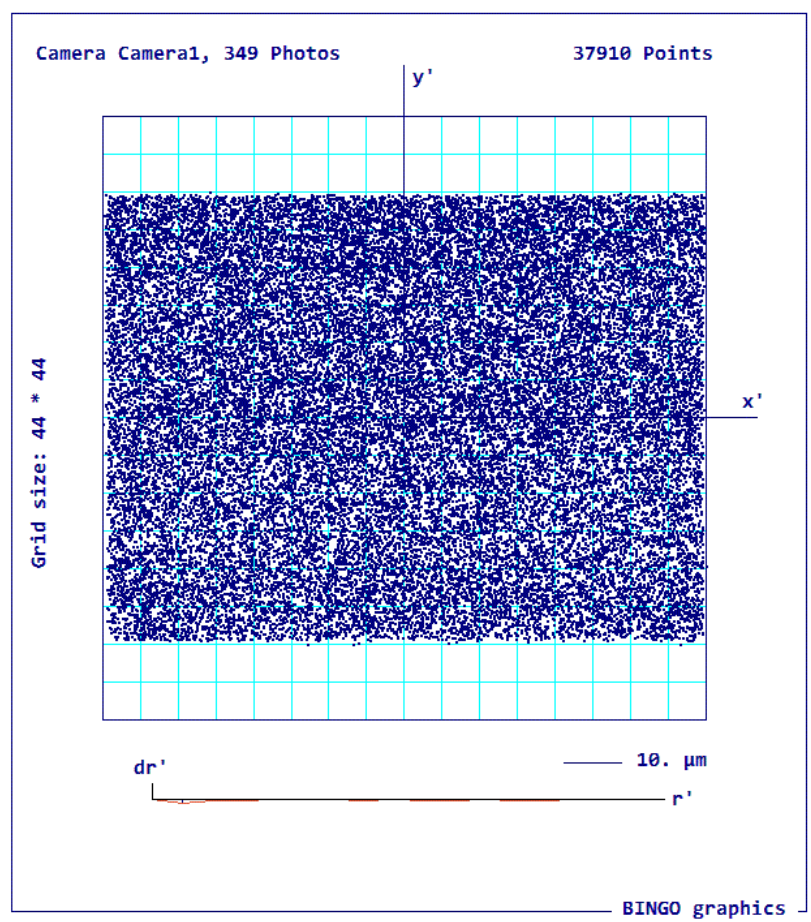

Figure 3: Point distribution of a selected camera, all points are projected into the image frame

After the flight mission with automatic method tie points were detected and the point measurements were imported into the adjustment. The full calibration process was made with BINGO and as a result, the adjusted point coordinates, camera parameters and the calibration grid for further processing was received.

\section{THEORY OF THE OBLIQUE CALIBRATION}

Our recent researches already showed us in theory the advantages of the oblique systems for calibration the focal length (Reference). In that theoretical test oblique geometry and camera data has been defined in a simulation project. In the test project the camera directions were defined with an angle to the nadir line of 12.5 degrees. In this case the models from the opposite directional flight lines show an angle of 25 degrees to each other. In the simulation a completely flat surface has been defined. The normal equation (1) provides a final result without any rank-defect. It means, that the selected geometric arrangement for the calibration is appropriate, otherwise the focal length and the principal point could not be determined.

$$
\text { Normal equation } \mathbf{A}^{\mathbf{T}} \mathbf{P} \mathbf{A x}=\mathbf{A}^{\mathbf{T}} \mathbf{P I}
$$

In this practical project we have an angle between the camera directions more than 25 degrees (it is about 80-90 degrees), so the mathematical requirement is fulfilled. With this demo project we want to prove the result of our former research.

\section{LENS DISTORTIONS CALIBRATION}

First we have calculated an initial orientation correction and a radial correction with help of the nadir camera and make use of the GPS/IMU airborne orientation. Because of the better overlap of the nadir camera images, it was the logical and right decision to start using these images first.

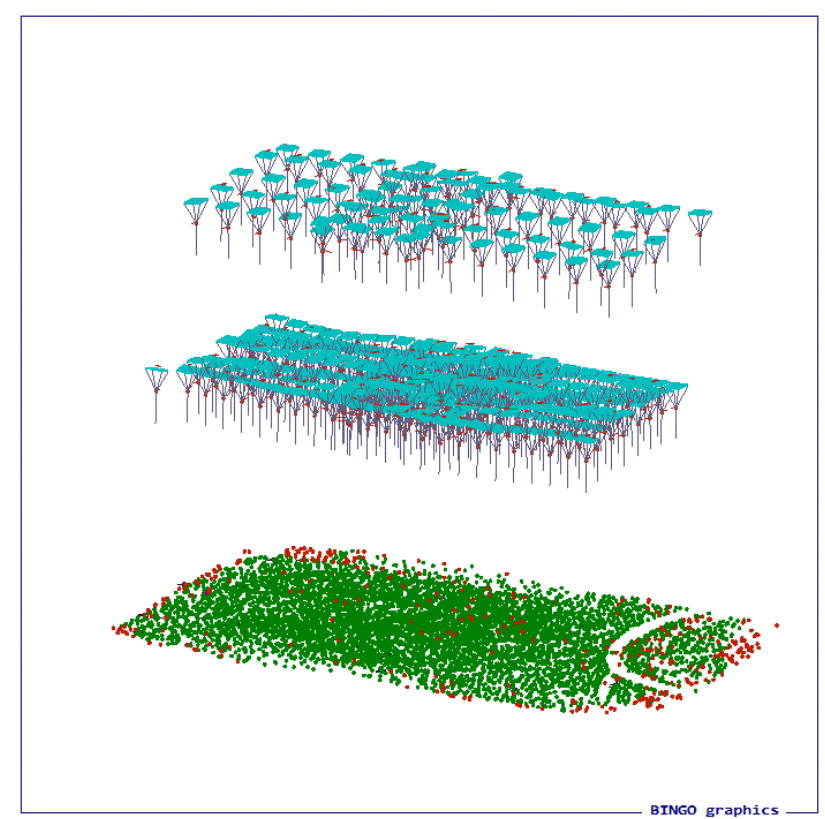

After the very first trials the radial remaining effects were the most dominant which needed to be corrected already at the starting phase. They were corrected with some general radial parameters.

After the preliminary radial correction and calculation of the initial camera positions, it was possible to turn on the oblique cameras as well. They were activated in the project step by step, always one new camera with all the 351 images. The stepwise project solution is a big advantage at the gross error detection.

In the next stage already all 5 cameras and the initial radial correction have been included as a correction. At this stage it was already possible to see the rigid geometry of the system, but smaller systematic effects could still be seen in the image surfaces through the remaining errors.

$$
\text { where } \quad \begin{aligned}
& \mathrm{A}=\text { model matrix } \\
& \mathrm{P}=\text { weight matrix } \\
& \mathrm{l}=\text { vector of obsevation } \\
& \mathrm{X}=\text { vector of unknown parameters }
\end{aligned}
$$



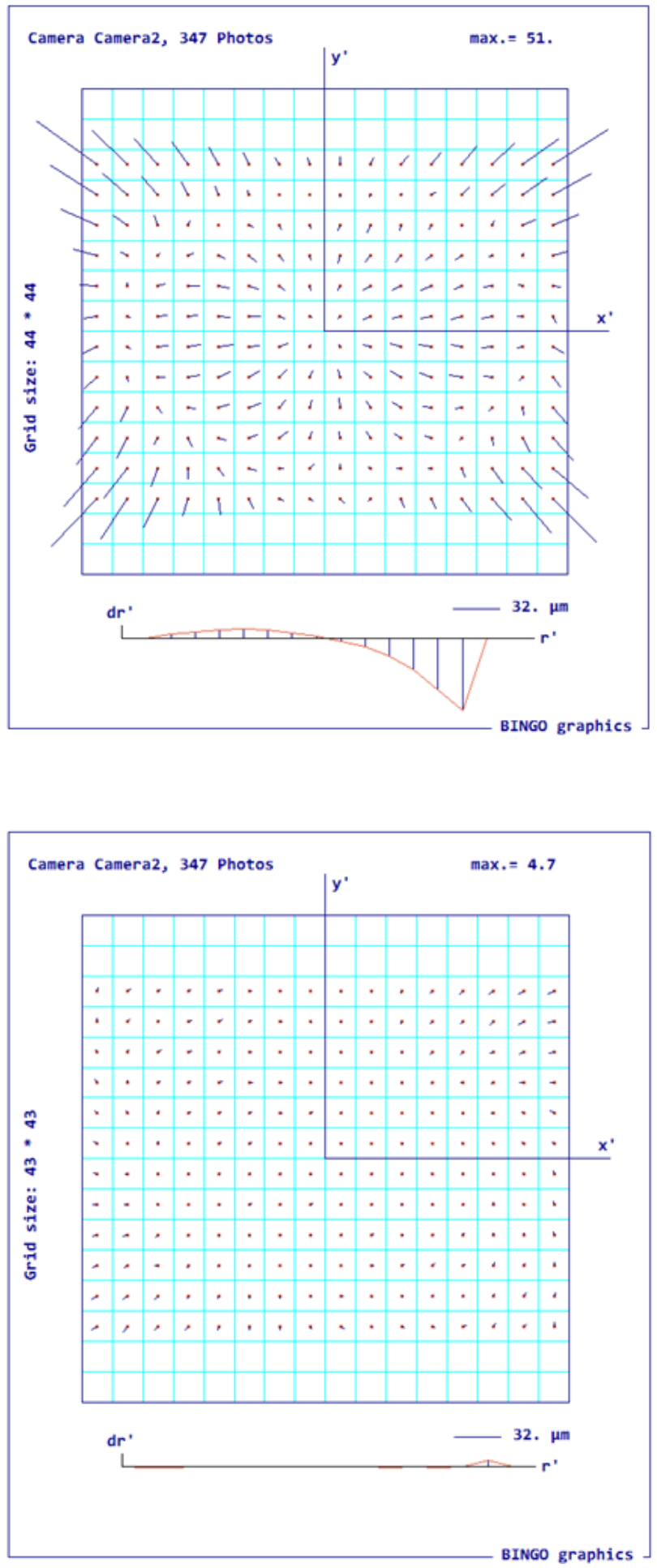

Figure 4. Remaining radial systematic effects before/after

At the above stage further parameters were also included, which were individually selected for every camera by analyzing the remaining errors in the Implo program of BINGO.

During this step two special parameters for medium format cameras and a parameter - which was developed especially for that camera type - were used.

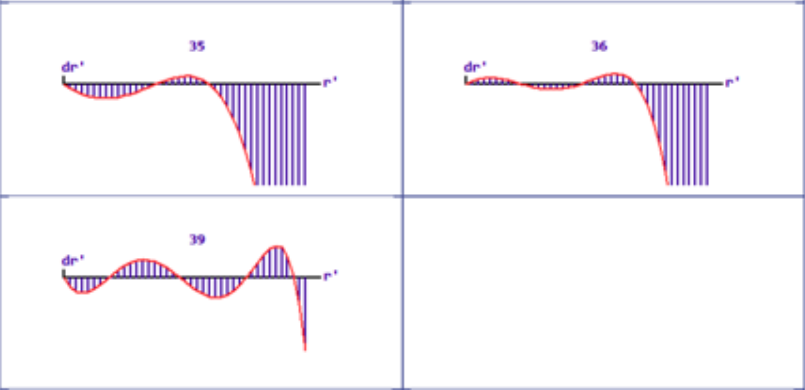

\section{CALIBRATION OF INTERIOR ELEMENTS WITH OBLIQUE SETTING}

After using the additional parameters there were still a few remaining systematic effects in the images. This is why it was necessary to check the camera parameters, like camera constant and principal points. First the PPA has been calibrated and the accuracy of the system increase remarkable.

With help of the oblique geometry the calibration of the camera constants were done. The camera directions have a bigger angle, that way the definition of the camera constant was possible.

For the calibration we have used all the cameras at the same time to reach better overlapping. The entire project is more stable and we were able to determine the focal length with higher accuracy.

All existing parameters and unknowns were activated on in iterative steps. This way we have been able to specify the correct weighting. After all, when all the variance components show a correct value for every measurement group the result will be considered as final.

The diagram below shows the remaining errors in the image space.

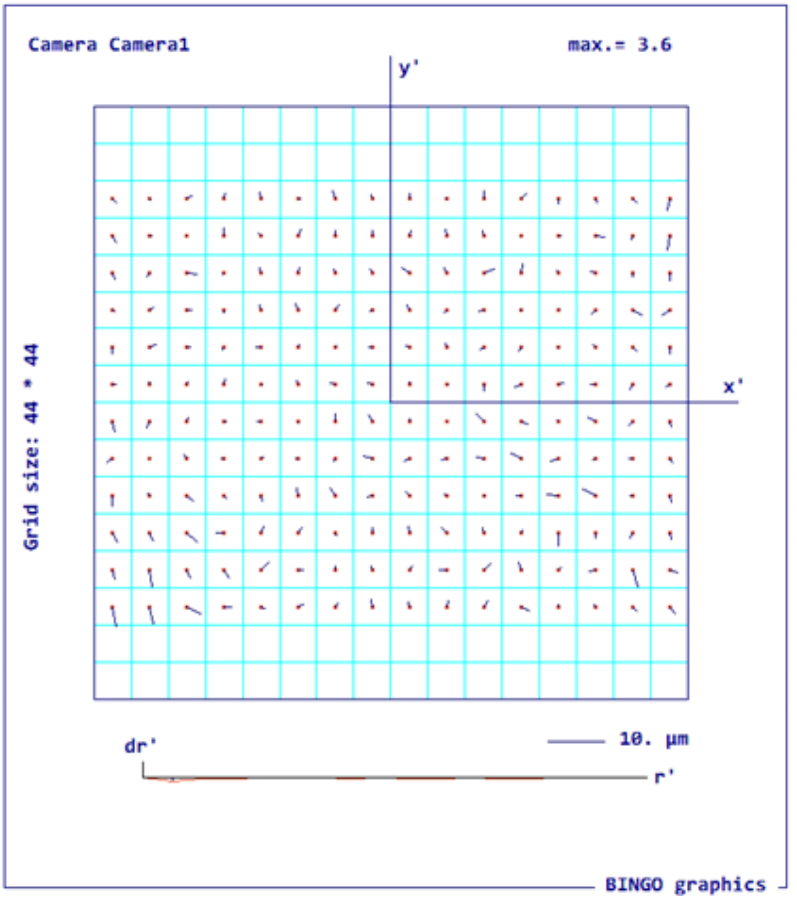

Figure 5. The remaining errors and their directions can be seen on a selected camera. 


\section{RESULTS}

Oblique cameras generate a huge number of images and a big variation of overlaps between the images. With modern AT software it is easier to measure tie-points in all images, in any case better than the process of co-registration.

At the camera calibration the oblique geometry brings a huge stability into the project, much better than a nadir camera alone can ever support. The camera constant can be determined in normal aerial projects just in case of special geometrical conditions. In an oblique project it is working stable and accurate in every case. In this practical case study we were able to verify what the theoretical results showed us earlier; proving that this is the way to build a more rigid and accurate camera system.

Dealing with the leverarms, camera-offsets and also the huge amount of images makes the handling a bit more complex, but the results analyzed by geometric aspects are outstanding.

Indeed there are many possibilities for errors, but actual softwares can easily detect if something is not set-up correctly.

In addition to stable camera-calibration and the very accurate aero-triangulation, the results of determining height points are far better than with only a nadir camera. Oblique cameras also support data for true orthophoto processing to fill gaps created by the nadir camera and, that is the final intention of the Oblique cameras, it enables the generation of a well textured 3D city model.

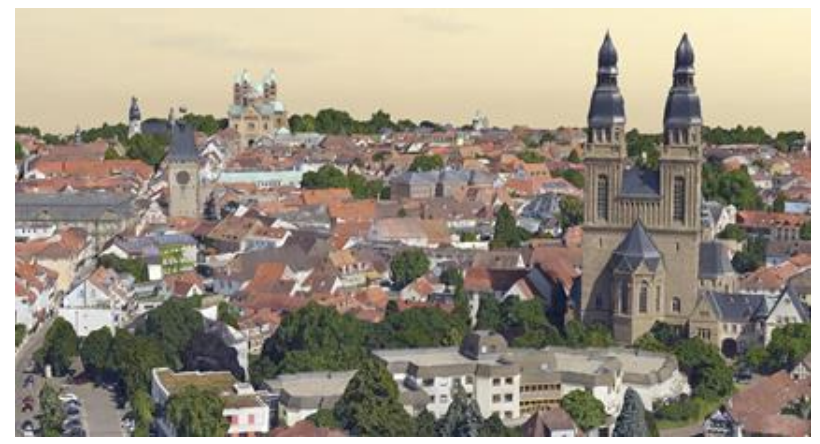

Figure 6 . Sreenshot of the resulted texturated 3D city model of the calibration area

\section{REFERENCES}

Kemper, G., Li Hongbo, Pauly, K. † (2008): New airborne Sensors and Platforms for specific applications in Photogrammetry and remote sensing; Proceedings of the ISPRS Congress 2008 in Beijing

Kemper, G. (2010): Neue luftgestützte Sensoren und Plattformen für verschiedenste Aufgaben in der Fernerkundung. 3 Ländertagung der DGPF, OVG und SGPBF, Wien.

Kemper, G. (2012): New Airborne Sensors and Platforms for Solving Specific Tasks in Remote Sensing; XXII ISPRS Congress, ISPRS Proceedings, Melbourne/ Australia

Kemper, G., Pivnicka, F., Geissler, S. (2012): Calibration Procedures in Mid Format Camera Setups; XXII ISPRS Congress, ISPRS Proceedings, Melbourne/ Australia.
Kruck, E. and Melykuti, B.: Kalibrierung von Oblique- und UAV Kameras, Jahrestagung der Deutschen Gesellschaft für Photogrammetrie, Fernerkundung und Geoinformation, Hamburg, 2014

Kruck, E., 2006: Simultaneous Calibration of Digital Aerial Survey Cameras. EuroSDR Camera Calibration Workshop, Castelldefels, Spain 